\title{
Psychiatrie-Ausbildung für Nicht-Psychiater
}

Psychiatrische Störungen wurden in den letzten 50 Jahren dramatisch häufiger, obwohl sie dank nebenwirkungsärmeren Psychopharmaka und dank spezifischeren Psychotherapien besser behandelbar geworden sind. Dies gilt besonders für Depressionen und Angststörungen. Studien zeigen, dass trotz des medialen Depressionsreigens (Stichwort Burn-out, Suizide von Fußballern) nur etwa jeder 3. depressive Patient erkannt und nur etwa jeder 15. (!) wenigstens 4 Wochen lang state-of-the art behandelt wurde; von der in allen Konsensuskonferenzen empfohlenen Psychotherapie ganz zu schweigen. Diese sollte ja nach übereinstimmender Meinung aller Fachleute bei Depressionen immer, bei leichten Depressionen sogar ausschließlich, angewandt werden.

Tatsächlich könnten die vielen PatientInnen mit Depressionen gar nicht alle von PsychiaterInnen fachgerecht behandelt werden, weil es schlicht zu viele sind. Die unsachgemäßen und teils gefährlich falschen Behandlungen werden überwiegend von Nicht-Psychiatern also Internisten, Allgemeinmedizinern, Gynäkologen, HNO-Ärzten, etc., also Ärzten, die mit der körperlichen Seite unserer Diagnosen besonders häufig in Kontakt kommen, durchgeführt. Manche ziehen keinen Fachmann bei, weil es keinen in der Nähe gibt. Die meisten trauen sich aber gar nicht, einem ihrer Patienten zuzumuten, zum Psychiater geschickt zu werden.

Es wäre dringend notwendig diese große Kollegengruppe besser psychiatrisch, psychosozial und psychotherapeutisch auszubilden. Es schadet unseren Patienten, dass Psychiatrie nicht verpflichtend im Turnus und den meisten Fachausbildungen vorgesehen ist. Turnusärzte werden viele Monate als SystemStudien zeigen, dass trotz des medialen Depressionsreigens (Stichwort Burn-out, Suizide von Fußballern) nur etwa jeder 3. depressive Patient erkannt und nur etwa jeder 15. (!) wenigstens 4 Wochen lang state-of-the art behandelt wurde; von der in allen Konsensuskonferenzen empfohlenen Psychotherapie ganz zu schweigen. erhalter auf Chirurgischen Abteilungen zum Assistieren und Administrieren und auf Internen Abteilungen zum Infusionen Anhängen und Administrieren gebraucht. Psychiatrie lernen sie nicht, obwohl sie in ihren Ordinationen später bei mindesten jedem 2. Patienten ein psychisches Problem zu erkennen und behandeln haben werden.

Suizid liegt nicht in der alleinigen Verantwortung eines betroffenen Menschen, sondern er ist auch ein erkennbarer und behandelbarer Zustand. Die Vorurteile gegen unser Fach werden weniger. Ich vermute, dass damit auch mehr Prozesse wegen Suiziden überwiegend gegen Nicht-Psychiater kommen werden. Muss erst das passieren, bevor die psychiatrische Weiterbildung wichtiger genommen wird.

Letzterer hat sich unser Journal mit Leib und Seele verschrieben. Ich danke hier für die zahlreicher werdenden Kasuistiken, die an uns herangetragen werden und bitte alle interessierten Kollegen darum, bei spannenden, unerwarteten, diagnostisch oder therapeutisch unklaren Fällen etc. eine Schilderung des Falls zum Zweck der Weiterbildung in der Psychopraxis in Betracht zu ziehen. In konkreten Fallberichten sollen aber auch typische state-of-the-art-Diagnosen und Therapien von Kollegen möglichst vieler Fachrichtungen geschildert werden.

Eine spannende Lektüre wünscht

Peter Fischer 